\title{
Successful management of atrio-esophageal fistula using a fully covered esophageal stent case report
}

\author{
Daisy Sanchez ${ }^{1}$, Francisco Tarrazzi ${ }^{1}$, Scott Harter ${ }^{2,3}$, Mark Block $^{1}$, Syed S. Razi ${ }^{1}$ \\ ${ }^{1}$ Division of Thoracic Surgery, Memorial Healthcare System, South Broward, FL, USA; ${ }^{2}$ Department of Anesthesiology, Memorial Healthcare \\ System, South Broward, FL, USA; ${ }^{3}$ Division of Thoracic Surgery, Department of Surgery, Memorial Healthcare System, South Broward, FL, USA \\ Correspondence to: Syed S. Razi, MD. Division of Thoracic Surgery, Memorial Healthcare System, 601 N Flamingo Rd., Ste 316, Pembroke Pines, FL \\ 3302, USA. Email: srazi@mhs.net.
}

\begin{abstract}
Atrio-esophageal fistula (AEF) is a rare but fatal complication of cardiac ablation procedures. Approaches to management have focused on prompt diagnosis and surgical correction, as there is a high risk of patient decompensation and death from cerebrovascular morbidity and mortality. Esophageal stenting is not a standard approach and has been used exclusively for palliation in select cases with limited outcomes data. We report a case of a 61-year-old male presenting in critical condition due to AEF 4 weeks after cardiac ablation for persistent atrial fibrillation. The patient presented in septic shock, multisystem organ failure and was not a candidate for definitive surgical repair. Therefore, he underwent treatment with covered esophageal stent placement. The post-operative course was complicated by septic shock and cranial emboli. Despite the complex presentation and clinical progression, the patient made a meaningful neurologic and physiologic recovery. At 8 weeks he was discharged and at 10 weeks the stent was removed with demonstration of complete healing of the AEF. While surgical repair remains the standard of care for treatment of AEF as a result of cardiac ablation, in high-risk patients, esophageal stenting may provide a means for effective palliation. Stenting may allow for spontaneous healing of AEF in these select high-risk populations.
\end{abstract}

Keywords: Case report; atrio-esophageal fistula (AEF); esophageal stent; cardiac ablation

Received: 07 June 2020; Accepted: 20 February 2021; Published: 10 July 2021.

doi: $10.21037 /$ shc-20-70

View this article at: http://dx.doi.org/10.21037/shc-20-70

\section{Introduction}

Atrio-esophageal fistula (AEF) is an exceedingly rare but usually fatal complication of atrial fibrillation ablation procedures (1). Due to its rarity and associated poor outcomes, there is no consensus approach to therapy. However, early diagnosis and intervention is considered essential for patient survival due to the increased risk of cerebrovascular events. In some cases, surgical correction is not feasible and therefore esophageal stenting has been used. Published results with the approach are very limited $(2,3)$. We present the following case in accordance with the CARE reporting checklist (available at http://dx.doi. org/10.21037/shc-20-70).

\section{Case presentation}

A 61-year-old male with a history of a bioprosthetic aortic valve replacement and coronary artery bypass graft underwent cardiac ablation for persistent post-operative atrial fibrillation. The procedure was uneventful and he was discharged home in a timely manner. One month later he presented to the emergency room with fever and lethargy (Figure 1). Workup revealed atrial fibrillation and sepsis with streptococcus mitis bacteremia. Pertinent laboratory results included a leukocytosis $\left(14 \times 10^{3} / \mu \mathrm{L}\right)$, lactic acidosis $(4.6 \mathrm{mmol} / \mathrm{L})$, an acute kidney injury [creatinine $(\mathrm{Cr}) /$ blood urea nitrogen (BUN), 1.5/34 mg/dL] and elevated liver function tests [alanine aminotransferase (ALT), 


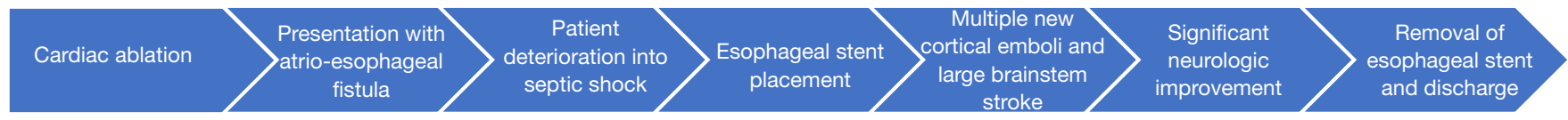

Figure 1 Timeline of treatment.

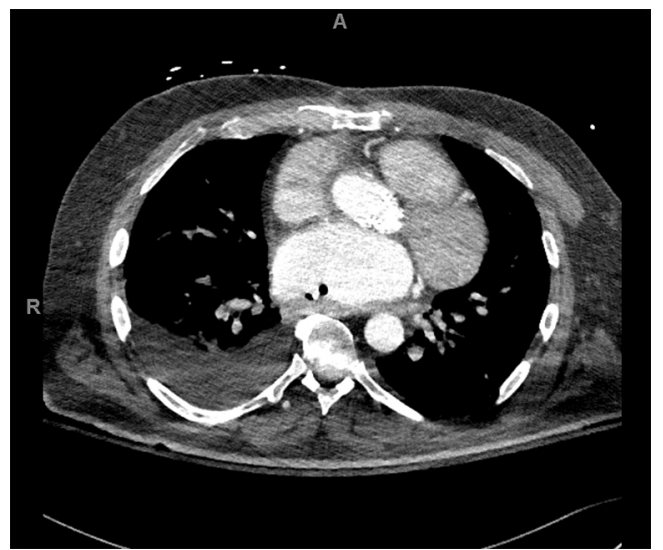

Figure 2 Computed tomography (CT) scan displaying a right sided pleural effusion and air in the left atrium.

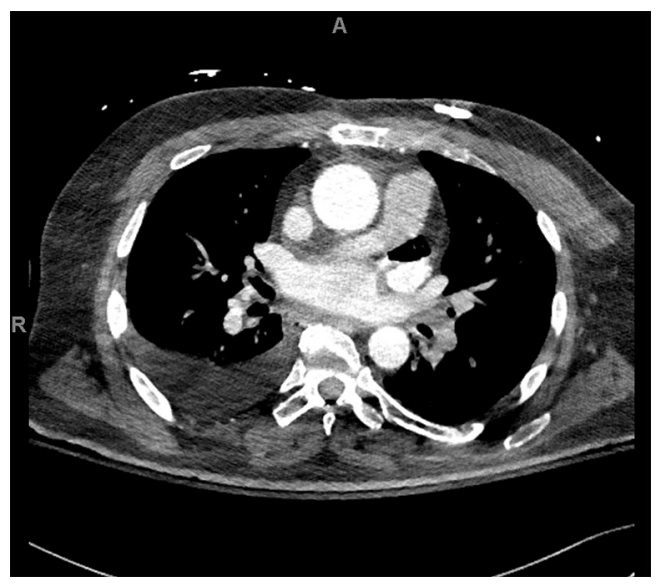

Figure 3 Computed tomography (CT) scan displaying a right pleural effusion and significant air in the left ventricle.

995 units/L and aspartate aminotransferase (AST), 1,686 units/L]. Computed tomography (CT) scan demonstrated pockets of air extending from the mid-thoracic esophagus into the left atrial appendage and left ventricle (Figures 2,3), suggestive of AEF. Head CT showed left frontal lobe petechial hyper densities consistent with embolic (septic) strokes.

The patient deteriorated rapidly with development of septic shock and multiorgan failure requiring maximal vasopressor support. He was then transferred to our facility for further management. While continuing the resuscitative efforts, a multi-disciplinary meeting was held and input obtained from cardiac and thoracic surgery as well as intensive care teams. Given the recent stroke and technical challenges of access to the posterior wall of the atrium after aortic valve replacement, the decision was made to defer definitive repair of the AEF. An alternative strategy was devised using fully covered esophageal stent placement for palliation.

In the operating room, upper gastrointestinal endoscopy was performed using the Olympus gastroscope (Olympus GIF-HQ190) with carbon dioxide $\left(\mathrm{CO}_{2}\right)$ insufflation. Despite minimal insufflation we encountered rapid hemodynamic collapse, secondary to AEF and $\mathrm{CO}_{2}$ embolization. Hence, the site of the AEF could not be examined. After resuscitating the patient, a 0.034 inflexible tip guidewire $(260 \mathrm{~cm})$ was advanced under fluoroscopic guidance and surface fiducial markers placed at intended proximal and distal sites to accommodate a $15-\mathrm{cm}$ fully covered esophageal stent from the carina to the gastroesophageal junction (Figure 4). The Merit Endotek fully covered esophageal stent $(18 \mathrm{~mm} \times 30 \mathrm{~mm})$ was successfully deployed under fluoroscopy and a nasogastric tube was secured for gastric decompression.

Post-operatively, head CT scan showed multiple new cortical emboli and a large brainstem pontine stoke. In the ensuing weeks, patient was treated with broad spectrum antibiotics (ceftriaxone, ampicillin/sulbactam and metronidazole) and underwent surgical placement of a jejunostomy tube for enteral feeding access. His septicemia resolved and he was successfully extubated 2 weeks following stent placement. Initial neurologic exam revealed dense left hemiparesis. His further hospital course was protracted. $\mathrm{He}$ received aggressive daily physical, occupational and speech therapy. Imaging during the hospitalization revealed slight 
CT scan at presentation

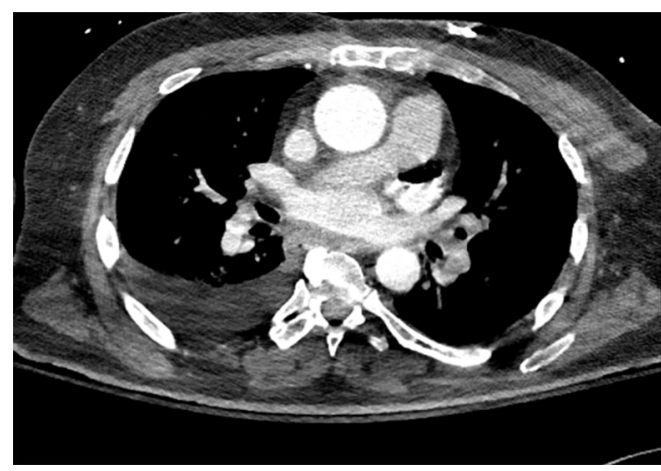

CT scan post esophageal stenting and removal

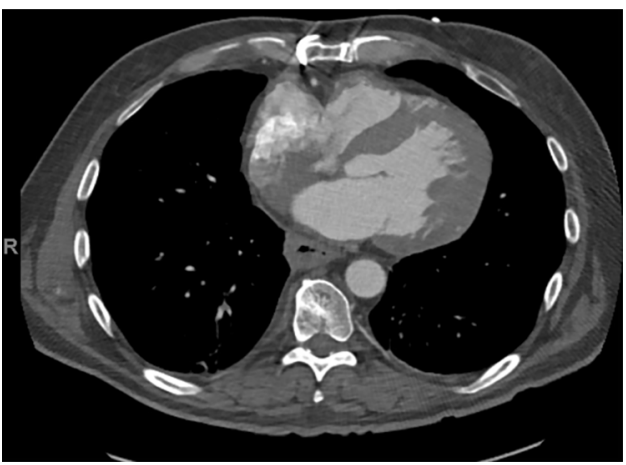

Figure 4 Before and after esophageal stenting. CT, computed tomography.

distal migration of the esophageal stent, but he did not show any sign of recurrent septicemia and hence intervention was deferred. He made significant neurologic recovery with the ability to hold simple conversations and ambulate with assistance. Eight weeks following stent placement he was discharged to an acute rehabilitation facility.

During the 10th week, he was readmitted to the hospital with a clogged jejunostomy feeding tube. He continued to recover neurological function and did not show any further evidence of recurrent neurological events or bacteremia. Repeat CT scan of the chest and contrast esophagogram did not show any fistulous communication between the esophagus and left atrium. In the process of contemplating definitive surgical repair, a repeat endoscopy was planned to replace the esophageal stent and re-evaluate the site of the fistula. In the operating room, usual preparation was undertaken for aggressive resuscitative support, as needed. The Olympus gastroscope was advanced in the esophagus with minimal $\mathrm{CO}_{2}$ insufflation and the whole esophagus examined. No gross abnormality was found, nor any changes in hemodynamics observed. The stent was in position and well anchored in the distal esophagus. Using alligator graspers, the proximal suture loop was held and the stent was withdrawn along with the endoscope (Figure 1). The gastroscope was again advanced into the esophagus with minimal insufflation and the entire esophagus examined. No abnormality was found in the entirety of the esophageal lumen and the mucosa appeared intact. The patient remained hemodynamically stable throughout the procedure, and had no new neurologic deficits observed upon emergence from anesthesia. Postoperatively, he was kept nil per os (NPO) for 3 days with continued tube feeds. A CT scan with oral contrast was performed 3 days post-operatively and the esophagus was visualized without evidence of AEF. Neurological recovery continued and he was walking more than 85 meters with a walker, able to move all extremities and converse clearly. $\mathrm{He}$ was started on a clear liquid diet and eventually advanced to a soft diet without complications. He was subsequently discharged to a skilled nursing facility with instructions to continue a soft diet and cyclic tube feeds.

All procedures performed in studies involving human participants were in accordance with the ethical standards of the institutional and/or national research committee(s) and with the Helsinki Declaration (as revised in 2013). Written informed consent was obtained from the patient.

\section{Discussion}

Radiofrequency catheter ablation is the standard of care for the management of refractory atrial fibrillation. Complications with catheter ablation are rare; however, when encountered they can be quite consequential including cardiac perforation, pericardial effusion, cardiac tamponade and AEF (1). AEF is a typically fatal complication of atrial ablation and requires prompt diagnosis and management. Diagnosis relies heavily on patient symptomatology prompting work-up at the time of presentation. Fever, neurologic, or gastrointestinal symptoms within the 2-4 weeks following cardiac ablation should prompt further evaluation and suspicion for this critical complication. Currently, no consensus guidelines exist for the management of AEF secondary to left atrial ablation therapy (4). Surgical repair has been reported as the preferred definitive treatment. Surgical correction requires excision of the fistulous communication 
between the esophagus and the left atrium, and requires cardiopulmonary bypass. However, previous cardiac surgery especially in patients who present in extremis often poses prohibitive risks for surgical repair. We therefore used a covered esophageal stent in an attempt to control ongoing contamination from AEF while the patient's condition stabilized from acute sepsis and stroke.

In a review of the literature, outcomes following nonoperative management compared to definitive surgical repair of AEF have been dismal. Jehaludi and colleagues reported a review of 65 cases of AEF post-cardiac ablation and compared the outcomes between surgical and nonsurgical management (5). Survival rates were significantly higher following surgical repair $(79.4 \%, \mathrm{n}=34)$, as opposed to $6.5 \%, \mathrm{n}=31$ following non-surgical management $(\mathrm{P}<0.001)$. Among the non-surgically treated, 5 patients who underwent esophageal stenting died. Yousuf and colleagues also reported a small case series and literature review regarding the management of AEF following left atrial ablation (6). The authors reported mortality rates of $96 \%(n=23), 100 \%(n=8)$ and $33 \%(n=36)$ with medical management, stent placement and surgical intervention, respectively. Eitel and colleagues reported successful nonsurgical management of AEF with esophageal stenting and pericardial drainage in three patients (2). The authors reported successful closure of AEF on 45, 22 and 28 days post-stenting. Additionally, two patients had stent migration and were repositioned and anchored with clip placements. This case provides a unique case report presenting successful management of AEF in a critically ill patient. Limitations of this report include the presentation of a sole patient and the lack of evidence to suggest reproducibility. Further limitations of this patient's management include the inability to perform stent placement under appropriate $\mathrm{CO}_{2}$ insufflation due to the risk of hemodynamic collapse. However, this case will aid in providing a larger pool of evidence for the future consideration of stent use in very specific clinical scenarios.

The high rate of mortality in non-surgical management of AEF is likely multifactorial. Many patients present in a septic, hemodynamically unstable state predisposing them to poor outcomes, and precluding any definitive surgical repair. Esophageal instrumentation also poses significant risks of air embolism with subsequent cerebral infarcts, as was seen in our patient. Consideration must also be given to the implicit risks of esophageal stenting, such as stent migration, perforation and respiratory tract compression.
Esophageal stricture has also been reported in healed AEF, amenable to esophageal dilatation (2).

\section{Conclusions}

In conclusion, surgical repair is the optimal management of AEF secondary to cardiac ablative treatments. In high-risk patients however, esophageal stenting may provide effective palliation, allowing spontaneous healing of AEF. Regardless of treatment strategy (operative and/or non-operative) prompt detection and rapid treatment is critical to avoid fatal complications of AEF.

\section{Acknowledgments}

Funding: None.

\section{Footnote}

Reporting Checklist: The authors have completed the CARE reporting checklist. Available at http://dx.doi.org/10.21037/ shc-20-70

Conflicts of Interest: All authors have completed the ICMJE uniform disclosure form (available at http://dx.doi. org/10.21037/shc-20-70). The authors have no conflicts of interest to declare.

Ethical Statement: The authors are accountable for all aspects of the work in ensuring that questions related to the accuracy or integrity of any part of the work are appropriately investigated and resolved. All procedures performed in studies involving human participants were in accordance with the ethical standards of the institutional and/or national research committee(s) and with the Helsinki Declaration (as revised in 2013). Written informed consent was obtained from the patient.

Open Access Statement: This is an Open Access article distributed in accordance with the Creative Commons Attribution-NonCommercial-NoDerivs 4.0 International License (CC BY-NC-ND 4.0), which permits the noncommercial replication and distribution of the article with the strict proviso that no changes or edits are made and the original work is properly cited (including links to both the formal publication through the relevant DOI and the license). See: https://creativecommons.org/licenses/by-nc-nd/4.0/. 


\section{References}

1. Zhang P, Zhang YY, Ye Q, et al. Characteristics of Atrial Fibrillation Patients Suffering Esophageal Injury Caused by Ablation for Atrial Fibrillation. Sci Rep 2020;10:2751.

2. Eitel C, Rolf S, Zachäus M, et al. Successful nonsurgical treatment of esophagopericardial fistulas after atrial fibrillation catheter ablation: a case series. Circ Arrhythm Electrophysiol 2013;6:675-81.

3. Mohanty S. Outcomes of atrio-esophageal fistula following catheter ablation of atrial fibrillation treated with surgical repair versus esophageal stenting. J Cardiovasc

doi: $10.21037 /$ shc-20-70

Cite this article as: Sanchez D, Tarrazzi F, Harter S, Block M, Razi SS. Successful management of atrio-esophageal fistula using a fully covered esophageal stent case report. Shanghai Chest 2021;5:33.
Electrophysiol 2014;25:E6.

4. Han HC, Ha FJ, Sanders P, et al. Atrioesophageal Fistula: Clinical Presentation, Procedural Characteristics, Diagnostic Investigations, and Treatment Outcomes. Circ Arrhythm Electrophysiol 2017;10:e005579.

5. Jehaludi A, Heist EK, Giveans MR, et al. Retrospective review of 65 atrioesophageal fistulas post atrial fibrillation ablation. Indian Pacing Electrophysiol J 2018;18:100-7.

6. Yousuf T, Keshmiri H, Bulwa Z, et al. Management of Atrio-Esophageal Fistula Following Left Atrial Ablation. Cardiol Res 2016;7:36-45. 